\title{
9
}

\section{Is Powerful Causation an Internal Relation?}

\author{
David Yates
}

\subsection{Introduction}

Take internal relations to be all $R$ such that true predications of $R$ are made true by intrinsic, non-relational properties of their subjects, and external relations to be all the rest. If a relation $\mathrm{a} R \mathrm{~b}$ is internal in this sense then we can account for the truth of ' $\mathrm{a} R \mathrm{~b}$ ' in terms of the intrinsic properties of a and $\mathrm{b}$, without reifying $R$. Token internal relations are not instances of irreducibly relational polyadic properties; they are relational truths with non-relational truthmakers, hence reducible. If there are any irreducible relational states in the world, then they will be found among the external relations. It is not the case, in my view, that externality is sufficient for irreducibility. That a relational predication ' $a R b$ ' is not made true by the intrinsic properties of $a$ and $b$ does not imply that it is not made true by the intrinsic properties of some thing or things. ${ }^{1}$ Still, I think it reasonable to suppose that if a given relation is internal, then its instances do not involve genuinely relational properties. Assuming height to be intrinsic, given that Alf is $1.85 \mathrm{~m}$ tall and Bob is $1.75 \mathrm{~m}$ tall, 'Alf is $0.1 \mathrm{~m}$ taller than Bob' is made true by the intrinsic properties of Alf and Bob. We do not need to say in addition that the ordered pair (Alf, Bob) instantiates a relational property being $0.1 \mathrm{~m}$ taller than, and to posit such a property as truthmaker would be otiose.

Some neo-Aristotelians hope to show that causal relations are internal, thereby reducing such relations to intrinsic properties of their relata. ${ }^{2}$ Water, they say, has the intrinsic power to dissolve salt, salt the reciprocal power to be dissolved by

${ }^{1}$ See Ch.1.3 in this volume for elaboration.

2 See for instance the papers by Lowe (Ch. 6), Simons (Ch. 7), and Heil (Ch. 8), in this volume; and Heil (2012), ch. 6. 
water. The cause of the salt's dissolving in the water is the coming together of these reciprocal powers, the dissolving of the salt their mutual manifestation. If causation is the mutual manifestation of powers, which I assume here without argument, then causal relations are internal only if powers are intrinsic to their bearers. Those who defend the intrinsicality of powers typically point to the fact that powers need not manifest in order to be actual, concluding that being powerful does not require the existence of anything else. ${ }^{3}$ Powers are directed towards their manifestations, but do not ontologically depend upon them, and so are intrinsic to their bearers.

There is a type-token ambiguity, however, in the claim that powers do not depend on their manifestations. Although a token power does not depend on its being manifested in order to be actual, the type of which it is a token ontologically depends upon its manifestation-type, because powers are relationally individuated. ${ }^{4}$ This raises two problems for the proposed neo-Aristotelian reduction of causal relations. First, it places the idea that powers are intrinsic in tension with the Aristotelian commitment to immanent universals. Second, and more seriously, even if the first problem can be solved, the internality of causal relations is secured only at the expense of type-causal relations which cannot be seen as internal in the same sense, and which arguably must be reified.

\subsection{Powerful Causation as an Internal Relation}

Langton and Lewis treat intrinsic properties as those that cannot differ between duplicates, where $x$ and $y$ are duplicates iff they share all the same basic intrinsic properties, and $\mathrm{P}$ is basic intrinsic iff $\mathrm{P}$ is (i) non-disjunctive and contingent, and (ii) independent of accompaniment. Here, $\mathrm{P}$ is independent of accompaniment iff, possibly (a) there is a lonely $\mathrm{P}$, (b) there is a non-lonely $\mathrm{P},(\mathrm{c})$ there is a lonely non-P, (d) there is a non-lonely non-P. An object is lonely at a world iff there exist at its world no other contingent, wholly distinct objects. ${ }^{5}$ Intuitively, the idea is that basic intrinsic properties are those natural properties such that having or lacking them is independent of the existence or non-existence of anything else. The intrinsic properties of $x$ are its basic intrinsic properties plus whatever other contingent properties are in common to every possible basic intrinsic duplicate $y$

${ }^{3}$ Heil (2003); Molnar (2003).

${ }^{4}$ When, in what follows, I say that powers are relationally individuated, I mean only that they are at least in part individuated by their relations to each other. Whether or not they are fully so individuated does not matter for my purposes, and I explain why this is so in section 9.6.

${ }^{5}$ According to this definition, an object with proper parts can be lonely. The parts themselves, provided they do not overlap, will be accompanied by the others. 
of $x$. I need not endorse this proposal in full generality here. I shall appeal only to the following necessary condition on intrinsicality: if a property $P$ is intrinsic, then possibly, there exists a lonely $P .^{6}$ If the power to dissolve salt is an intrinsic property of this sample of water, then something could have this power in an otherwise empty world.

Internal relations are determined by the intrinsic properties of the relata. On the current conception of intrinsic properties as those that cannot differ between basic intrinsic duplicates, internal relations are most naturally conceived as those that cannot differ between ordered $\mathrm{n}$-tuples of basic intrinsic duplicates. Pick any $\mathrm{n}$-ary relation $\mathrm{R}$ you like, and any ordered $\mathrm{n}$-tuple $\left(x_{1}, \ldots, x_{\mathrm{n}}\right)$. If $\mathrm{R}$ is an internal relation, then $\mathrm{R}\left(x_{1}, \ldots, x_{\mathrm{n}}\right)$ iff necessarily, for any ordered $\mathrm{n}$-tuple $\left(y_{1}, \ldots, y_{\mathrm{n}}\right)$ such that for any $i, \mathrm{y}_{i}$ is a basic intrinsic duplicate of $x_{i}, \mathrm{R}\left(y_{1}, \ldots, y_{\mathrm{n}}\right)$. Duplicate the relata as to all natural properties that are independent of accompaniment, and you thereby duplicate any internal relations in which the elements stand. A consequence of this view-on the assumption that relations that hold in virtue of basic intrinsic properties of the relata are themselves natural properties-is that internal relations are also intrinsic to their relata, since they can hold, or fail to hold, independently of accompaniment, and cannot differ between basic intrinsic duplicates. ${ }^{7}$

Several other extant accounts agree that the possibility of loneliness is necessary for intrinsicality. Vallentyne, for instance, argues that intrinsic properties are those an object $x$ would retain if everything else (objects distinct from $x$, and even regions of spacetime not occupied by $x$ ) were subtracted from the world. ${ }^{8}$ Vallentyne analyses intrinsicality not in terms of properties possessed by all possible duplicates of $x$, but in terms of properties $x$ itself would retain if everything else were taken away. ${ }^{9}$ This account implies that if $\mathrm{P}$ is intrinsic to $x$, then there could be a lonely $\mathrm{P}-x$ itself, once everything that is not $x$ has been removed. Francescotti analyses intrinsicality in terms of non-relationalityroughly, properties intrinsic to $x$ are those properties $x$ has but does not have in virtue of standing in a relation to any object distinct from $x$, and intrinsic

${ }^{6}$ I will question the sufficiency of the above conditions for intrinsicality in section 9.5.

7 See Langton and Lewis (1998), pp. 343-4 for a more detailed argument. As Langton and Lewis point out, it does not follow that all intrinsic relations are internal. Spatiotemporal relations are intrinsic, in that they are natural and independent of accompaniment, but they are not obviously internal. See Lowe (Ch. 6 in this volume) for an argument for the internality of spatiotemporal relations.

${ }^{8}$ Vallentyne (1997).

9 For this reason, Vallentyne requires transworld identity of $x$ and so cannot endorse Lewis' counterpart-theoretic treatment of modality. Further discussion is beyond the scope of the present work; see Yablo (1999). 
properties simpliciter are those that are intrinsic to anything that has them. ${ }^{10}$ If only accompanied objects can have some property $\mathrm{P}$, then presumably Ps have $\mathrm{P}$ partly in virtue of being accompanied, so $\mathrm{P}$ will not come out intrinsic. Conversely, if $\mathrm{P}$ is intrinsic, then Ps need not be accompanied. Witmer, Butchard, and Trogdon endorse a version of Langton and Lewis's account that dispenses with the appeal to naturalness. They argue that an object $x$ has $\mathrm{P}$ in an intrinsic fashion iff (i) $\mathrm{P}$ is independent of accompaniment, and (ii) for any property $\mathrm{Q}$, if $x$ has $\mathrm{P}$ in virtue of having $\mathrm{Q}$, then $\mathrm{Q}$ is also independent of accompaniment. A property $\mathrm{P}$ is intrinsic iff, necessarily, if $x$ has $\mathrm{P}$, then $x$ has $\mathrm{P}$ in an intrinsic fashion. ${ }^{11}$ Once more, if $\mathrm{P}$ is intrinsic, then possibly, there is a lonely $\mathrm{P}$. The reason for this consensus on the possibility of loneliness is, I take it, that intrinsic properties are thought of as those that characterize the way the bearer is, in itself, regardless of how it relates to anything else. ${ }^{12}$

If powers are intrinsic, then powerful causation is an internal relation, although this is not immediately obvious; consider again the example of water dissolving salt. The water has the power to dissolve the salt, and the salt has the reciprocal power to be dissolved by the water. When the two are brought together, these powers mutually manifest as the dissolving of the salt in the water. But does this not involve spatiotemporal relations? The salt must be immersed in the water in order for the powers to manifest; many (if not all) powers appear to have spatiotemporal manifestation conditions. Unless spatiotemporal relations themselves are internal, it seems powerful causation will be an external relation, but this is not so. The cause, according to those who endorse powerful causation, is neither the salt nor the water, but the salt's immersion in the water; the effect is the dissolving of the salt. Internal relations hold in virtue of the basic intrinsic properties of the relata, and the property of being an immersion of salt in water is plausibly a basic intrinsic property of a complex cause. Nothing in our current conception of internal relations or intrinsic properties precludes the relata being intrinsically complex, and having-intrinsically-certain spatiotemporal relations between their proper parts. For causal relations to come out internal, the cause relatum must be taken to involve the interaction of reciprocal powers, which includes their spatiotemporal relatedness.

Mumford and Anjum hold that causes do not necessitate their effects, because it is possible for preventers to interfere with the manifestation of powers. ${ }^{13}$ At

${ }^{10}$ Francescotti (1999). $\quad{ }^{11}$ Witmer, Butchard, and Trogdon (2005).

12 It is interesting to note that all three accounts described in the preceding paragraph entail that a property $\mathrm{P}$ is intrinsic only if it is possible for any of its bearers to have it if lonely. I will return to this point in section 9.5, when I question the sufficiency of the Langton-Lewis analysis.

13 Mumford and Anjum (2011), esp. ch. 3. 
first blush, it may seem as though the above conception of causation as an internal relation requires that causes do necessitate their effects, since I hold that internal relations could not possibly fail to hold between basic intrinsic duplicates of the relata. However, suppose we mask the reciprocal dispositions of the salt and water, perhaps by wrapping the salt in watertight plastic. Or again, suppose-to use Mumford and Anjum's example-that a match is struck in such a way that all factors required for it to light are present, but a sudden gust of wind prevents its lighting. Mumford and Anjum argue for an 'antecedent strengthening' test of necessitation: if A necessitates B, then for any $\phi$, if $\mathrm{A}$ and $\phi$, then B. Causation fails this test, since any cause can occur without its effect if an appropriate preventer occurs.

I need not take issue with Mumford and Anjum's claim that such examples show that causes do not necessitate their effects. I am concerned only with whether they show that powerful causation is external. Being wrapped in plastic is an extrinsic property of the salt; being struck during a sudden gust of wind is an extrinsic property of the match. But now it seems that not every basic intrinsic duplicate of the salt will dissolve in the water, and not every basic intrinsic duplicate of the match will light when struck. A tempting thought, but, I think, a mistake. Focus on the salt and water example. As I have already explained, the dissolving of the salt by the water is an internal causal relation only if we take the cause to be the immersion of the salt in the water, holding fixed, of course, that the water has the power to dissolve the salt and the salt the corresponding reciprocal power. Wrapping the salt in plastic changes the basic intrinsic properties of the cause, and so is no counterexample to the internality of causal relations.

What I am claiming here is that an extrinsic property of the salt itself-being wrapped in plastic, which it could not have if it were lonely-will count as an intrinsic property of the relevant cause relatum, the salt's being immersed in the water. The same goes for the gust of wind which would have prevented the lighting of the match-extrinsic to the striking of the match, which may occur in many different ways, but intrinsic to the cause event, which will include all the powers whose mutual manifestation on this occasion result in its lighting. As Heil notes, we can alter the ways in which powers manifest by adding further powers into the mix, but this does not mean that powerful causation is external, because the additional powers-the preventers, in Mumford and Anjum's terminologyalter the intrinsic properties of the relata. ${ }^{14}$ If we are careful enough to duplicate

14 Heil, 'Causal Relations', Ch. 8 in this volume. 
the basic intrinsic properties of salt and water, of match, matchbox, and atmosphere, then we are bound to be at a world where no preventers occur.

Note that those who take causal relations to be internal need not reject Mumford and Anjum's view that causes do not necessitate their effects. If we take events to be modally robust, then altering the intrinsic properties of a cause so as to prevent its effect need not prevent the occurrence of the cause. However, if antecedent strengthening fails only in cases where preventers alter the intrinsic properties of causes, the failure of the prevented effect to occur does not count against the internality of the causal relation. Internality does not require that causes necessitate their effects, but only that the occurrence of cause and effect in a particular way necessitates that they are related as cause and effect.

\subsection{Are Powers Intrinsic?}

The question I address in this section is: if powers have relational essences, how can they be intrinsic? ${ }^{15}$ Powers are relationally individuated in two senses: it is in the nature of a given power that there are specific partner powers with which it must be combined in order to manifest, and each combination of partner powers has a specific manifestation type or types. ${ }^{16}$ The powers ontology is typically seen as a form of structuralism, in that powers are individuated by their places in a relational structure. ${ }^{17}$ Weaker forms of powers ontology, such as Heil's powerful qualities theory, also plausibly require that powers have at least partially relational essences. Powers, for Heil, are essentially related both to their partner powers, and the manifestation types that result from their various combinations. True, Heil thinks that powers are also qualities, in that they can be thought of (through 'partial consideration') in non-relational terms. ${ }^{18}$ However, it is not in virtue of our ways of thinking about powers that they are such as to combine with their partner powers to yield certain manifestations. As Heil himself says, "[i]n

\footnotetext{
${ }^{15}$ An anonymous referee points out to me that Tugby (2013) employs arguments similar to those found in this section to argue that dispositionalists must embrace Platonism about universals if they want to maintain that powers are intrinsic. The arguments of this paper were developed independently, and while I agree that Platonism is one way to secure the intrinsicality of powers, I do not think it is the only way.

${ }^{16}$ If all properties are powers, then this is double dependence on powers. I speak in what follows as if the manifestations of powers were instances of further powers, but nothing for present purposes turns on this.

17 Bird (2007a, b).

${ }^{18}$ Heil (2012), ch. 4 and (2003). Sphericality, for instance, can be characterized non-relationally-as a property satisfying a certain mathematical formula-or in terms of the way it combines with other powerful properties to yield manifestations such as rolling.
} 
virtue of being as it is, each power would manifest itself in a particular way with particular kinds of reciprocal partner." ${ }^{19}$ Having certain partner powers and manifestation types are part of the essential nature of each power. The power to dissolve salt is of its nature both directed towards the dissolution of salt as a manifestation type, and has the solubility of salt as a partner power. As Bird notes, that powers are relational in this sense does not imply that powers are relational properties of their bearers. Powers are second-order relational, but firstorder intrinsic-essentially related to other powers at type-level, but intrinsic to their bearers. ${ }^{20}$

The kind of dependence of powers on reciprocal powers and manifestations sketched above is second-order in the following sense: a given power depends on the existence of its reciprocal partner powers and its possible manifestation types, but not on its actually combining with the former to bring about the latter. I now give an example to clarify the sort of dependencies we see in physics. Consider the time-dependent Schrödinger equation for a single particle moving in a radial electromagnetic potential $V$ :

$$
i \hbar \frac{\partial \Psi(r, t)}{\partial t}=\hat{\mathrm{H}} \Psi(r, t)\left[\text { where } \hat{\mathrm{H}}=-\frac{\hbar^{2}}{2 m} \nabla^{2}+V(r)\right]
$$

$\Psi$ is the particle's wave function, interpreted physically as encoding a probability distribution. The term on the left hand side represents the total energy of the particle, and the two terms of the Hamiltonian operator $\hat{H}$ represent its kinetic and potential energies, in turn. ${ }^{21}$ To derive $\hat{\mathrm{H}}$ for a particular case we need to specify the nature of the potential $V$ in which the particle moves. For instance, for an electron orbiting a positively charged nucleus of atomic number $Z$, we substitute the Coulomb potential for $V(r)$ to get: ${ }^{22}$

$$
i \hbar \frac{\partial \Psi(r, t)}{\partial t}=\left(-\frac{\hbar^{2}}{2 m} \nabla^{2}-\frac{k_{e} z e^{2}}{r}\right) \Psi(r, t)
$$

Once $\hat{\mathrm{H}}$ and appropriate boundary conditions are specified, we can solve for $\Psi$ and use the Born rule to calculate the probability distribution over various measurement outcomes (position, momentum, etc.). Now suppose that fundamental physical properties are powers, and that which powers they are is fully described by completed physical science. This being the case, we can read off the

${ }^{19}$ Heil (2012), p. $75 . \quad{ }^{20} \operatorname{Bird}(2007 \mathrm{~b})$.

${ }^{21} \nabla^{2} \Psi=\left(\partial^{2} / \partial x^{2}+\partial^{2} / \partial y^{2}+\partial^{2} / \partial z^{2}\right) \Psi$. The details are unimportant here.

${ }^{22}$ The Coulomb potential $V$ between two point charges $q_{1}$ and $q_{2}$ is given by $V=k_{e} q_{1} q_{2} / r$, where $k_{e}$ is Coulomb's constant. Again, details are unimportant for present purposes. 
ontological dependencies from the appropriate equations. Write down the fundamental laws involving a given property P. P ontologically depends on any property $\mathrm{Q}$ that you cannot but refer to in framing those laws, and any properties upon which Q depends in this manner. If the Schrödinger equation above (at least partially) specifies which powers mass and charge are, then mass and charge are mutually ontologically dependent. They are interdefined in terms of the way in which an electromagnetic potential, generated by a charge, alters the wave function of a particle with a certain mass and kinetic energy. In order to specify which power mass is, we must refer to charge, and vice versa. It follows that neither mass nor charge could be the power it is unless the other also existed.

This immediately raises the worry that powers cannot be intrinsic if we suppose, with Aristotle, that powers are immanent: that for a power to exist at a world, there must be at least one thing in the history of that world that has it. If the identity of mass, qua power, is (partially) described by a law relating it (inter alia) to charge, and this law requires the existence of charge as a partial truthmaker, and the existence of a property at a world requires that something there has it, then for anything to be massive, something must be charged. The converse is of course also true. If our current conception of intrinsic properties is correct, then a property $\mathrm{P}$ is intrinsic only if there is a lonely $\mathrm{P}$. For Aristotelians, however, it seems there will not be any lonely empowered particulars at all, assuming every power ontologically depends upon at least one other. For anything to possess a given power, there must exist at its world bearers of all the other powers involved in its relational individuation. ${ }^{23}$

Let us take stock. If powers are ontologically dependent on each other, then the existence of a power at a world requires the existence of those powers upon which it depends. But for Aristotelians, a power exists at a world only if it has instances at that world, so for any $x$ to possess a power $P$, there must exist bearers of all powers upon which $\mathrm{P}$ depends. But in that case $\mathrm{P}$ is not intrinsic, because $\mathrm{Ps}$ are not possibly lonely. Think of it this way: Aristotelians cannot help themselves to Bird's idea that powers are second-order relational but first-order intrinsic, because the secondorder relations cannot obtain without first-order relata. There are two broad strategies available at this point: (A) reject relational individuation, keeping hold of the Aristotelian commitment to immanent universals; (B) keep relational individuation, and either reject immanence, or find a way to make relational individuation,

\footnotetext{
${ }^{23}$ Nothing I have said thus far suggests that powers are relational properties, and I do not think they are. My point thus far has merely been to raise doubts concerning the intrinsicality of immanent powers, stemming from the supposition that they are relationally individuated. I shall have more to say about this presently.
} 
immanence and intrinsicality compatible. Let us now consider these strategies in turn, starting with the idea that powers might be somehow self-contained.

\subsection{Self-Contained Powers}

Some deny that powers are relationally individuated, and if they are not, then neither are they ontologically dependent upon each other. Marmodoro, for instance, claims that the manifestation of a power is a distinct state of the power itself, rather than a numerically distinct property. ${ }^{24}$ Powers so conceived ontologically depend only upon their reciprocal partner powers for their identities-upon which powers they must be combined with to get them from potentiality to activation. In some cases, however, it seems that the partner powers are just further instances of the same power in potentiality. Suppose we say that the power to heat is activated by the heating body possessing greater heat than the heated body, and that its activation consists in its heating that body. This does not seem to involve any powers other than heat itself. I do not think this theory is plausible for heat when one considers the physical mechanisms that realize heating, but will not argue the point here, because I think it is perfectly clear that at least some fundamental powers have numerically distinct properties as their manifestation types.

For simplicity, let us think in terms of classical mechanics, in which the exertion of a given force accelerates a body at a rate determined by its inertial mass. Suppose we say that the essence of charge consists in generating an electric field, and the electric field generated by a given charge is simply a measure of the mutual force it and a unit charge would exert upon each other. Now it appears (reifying forces for the sake of argument) that we may conceive of charge as the power to exert a force on other charged particles, where (i) the exertion of such a force is charge in its activated state rather than in potentiality, and (ii) the reciprocal partner power of charge is another charged particle at a certain separation. No properties other than charge seem to be required to specify the essential nature of charge. This view seems plausible until we ask: what is it to exert a force? If charge is the power to exert forces on other charges, where the exertion of such a force is charge in its activated state, and force derives its identity (at least partially) from the acceleration it produces per unit mass, then charge in its activated state ontologically depends upon mass. ${ }^{25}$

${ }^{24}$ See Marmodoro (forthcoming a).

25 This point is not restricted to classical mechanics. The time-dependent Schrödinger equation discussed in section 9.3 can be seen in the same light, with the effect of a Coulomb potential on the wave function of a charged particle likewise dependent upon the mass of the particle. 
Something has gone wrong. We wanted to treat charge as a power that comes in (at least) two states: in potentiality, and exerting a Coulomb force, but when in the second state, charge accelerates the bodies upon which it exerts a force, at a rate depending (inter alia) on their masses. This dependency suggests that the manifestation of charge cannot be an activated state of charge itself. It is far more plausible to hold that mass, charge, and force are distinct reciprocal partner powers among whose mutual manifestation types are accelerations. There is simply nothing we can plausibly identify as the self-contained manifestation type of each partner power, when taken individually. Note that this argument does not depend on reification of forces, and is in fact clearer without it. If forces are fictions, then the only candidate manifestations of charge in cases of classical Coulombic interactions are the accelerations of charged particles, which cannot be regarded as activated states of charge.

Perhaps there is another way for powers to be self-contained. Recall Alf and Bob, who are $1.85 \mathrm{~m}$ and $1.75 \mathrm{~m}$ tall respectively, but suppose in addition that they have their heights essentially. Suppose further-uncontroversially-that all essential properties are necessary. It follows that wherever Alf and Bob both exist, Alf is $0.1 \mathrm{~m}$ taller than Bob. Alf and Bob could each exist without the other, but wherever both exist, they stand in a specific relation, in virtue of their essential intrinsic properties. Interestingly, the same is arguably true of certain properties. Let us suppose for the sake of argument that sphericality is the property of being an $x$ such that all points on $x$ 's surface are equidistant from a fixed point. In virtue of having this property, spheres are thus-and-so disposed-the sphericality of a rigid material body contributes to its disposition to roll down an inclined plane in a gravitational field, for instance. To echo Shoemaker, we might even describe sphericality as the power to roll conditionally on being massive, rigid, on an inclined plane and in a gravitational field. ${ }^{26}$

Here is the key point: assuming powers to be relationally individuated, then given immanent universals, sphericality cannot be this conditional power at worlds where mass, rigidity, and gravitation do not exist. Why not? Supposeagain for the sake of argument-that there is a possible world $\mathrm{W}$ containing no instances of mass or rigidity, and no gravitational fields, but at which there are massless bodies such that all points on their surfaces are equidistant from a fixed point. Arguing for this point in detail would take us too far afield, so let us simply agree with the intuition that such bodies are spherical. ${ }^{27}$ At $\mathrm{W}$ sphericality cannot

\footnotetext{
26 Shoemaker (1980).

27 This is harmless because I am appealing to sphericality for illustrative purposes only, and in any case do not endorse the theory of powers as self-contained which will emerge from the illustration.
} 
be the power to roll down an inclined plane in a gravitational field conditionally on being massive and rigid, because it is arguably not true at W that for any $x$, if $x$ were massive, rigid, and spherical, $x$ would roll down an inclined plane in a gravitational field. Some of the truthmakers of the counterfactuals that describe sphericality's causal contributions at our world are missing from W. If we want to say that sphericality is a conditional power around here, we had better say that it is only contingently, hence accidentally, the conditional power it actually is.

Perhaps this conclusion can be generalized to all powers. Here is how the theory might go. For Aristotelians to maintain that powers are intrinsic properties, and hence that powerful causation is an internal relation, they can hold that powers are accidentally the powers they are, and so are not ontologically dependent upon each other. Sphericality is the property of being an $x$ such that all points on $x$ 's surface are equidistant from a fixed point. At our world, because mass, rigidity, and the like exist, sphericality combines, in virtue of being the property it is, with those other properties, to manifest as rolling under certain circumstances. Sphericality, mass, and rigidity are such that wherever they all exist, things will roll. But it does not follow that any of them, considered in isolation from the others, is the power to do anything. Sphericality is a certain power at our world, we might say, partly in virtue of the other properties that exist around here. There are lonely powers in the sense that properties that are contingently powers around here can be instantiated by lonely particulars, and in such cases they fail to be the powers they are at our world-because they fail to be powers at any world.

It is easy to believe that sphericality is only accidentally a power, because the following claims are intuitively true: (i) we can capture its nature in nonrelational terms, (ii) we can do this in a way that makes it transparent why sphericality causally relates in the way it does to properties such as mass and rigidity. Unfortunately, we cannot do the same for physical properties such as mass and charge: our most fundamental characterizations of such properties describe them solely in terms of causal-nomic relations to each other. Perhaps mass, like sphericality, is a qualitative property which-in virtue of being the property it is-necessarily combines with other properties in a certain way. ${ }^{28}$

${ }^{28}$ Compare Jacobs' (2011) theory of fundamental properties as powerful qualities. Jacobs conceives such qualities as rich, non-mental qualitative natures, which, in virtue of being the qualitative natures they are, are partial truthmakers of certain counterfactuals (those, I take it, that are deducible from physical laws). The claim that fundamental properties such as mass and charge are qualitative counterfactual truthmakers, however, leaves open whether the qualities are (a) essentially powers and ontologically dependent upon each other, or (b) accidentally powers and ontologically independent. Jacobs does not say which. 
However, the two cases are importantly different. We can say which property sphericality is without reference to the properties with which it combines, at our world, to yield rolling. However, we are at a loss to say which property mass is without saying what it does. Physical science, as is by now familiar, conceives physical properties in purely structural terms, and that is precisely to conceive them in a way that undermines the view that they could be accidental powers. If physical powers are only accidentally the powers they are, then we cannot say which properties they are essentially. Mass, charge, and the like-if they are only accidentally powers-are essentially ineffable.

There are two further problems with the current theory. First, the idea that a property could be a power and yet have its causal role accidentally will seem to many to be a contradiction in terms. Sphericality has its causal role in virtue of being the property it is, and it arguably does not possess that role essentially, but this does not imply that sphericality is an accidental power, it implies that sphericality is not a power at all. Powers are individuated by their causal roles, and do not possess those roles in virtue of a prior, self-contained qualitative essence. Second, and relatedly, is that it is far from clear that accidental powers could stand in internal causal relations. It is certainly possible for individuals such as Alf and Bob to stand in internal relations in virtue of their essential natures, without thereby having relational essences, but this does not show that a set of self-contained properties could stand in internal causal relations solely in virtue of being the properties they are. In fact, the case of sphericality, to which I appealed to make the theory seem plausible, depends on the prior assumption that mass, for instance, already has its causal role. In virtue of what, though? Those who take mass to be a power will be inclined to say: in virtue of nothing at all, and that is why mass is a power, while sphericality is not. So much for the idea that powers might be thought of as self-contained properties, ontologically independent of each other.

\subsection{Relational Individuation, Immanence, and Intrinsicality}

Our problem is that Aristotelian immanence, given the relational individuation of powers, seems to imply that Aristotelian powers cannot be instantiated by lonely particulars, hence that they are not intrinsic. One option at this point is to embrace a transcendental conception of powers. Amongst those who endorse ontologies of powers, some are more sympathetic to Platonism than others. Bird, for instance, thinks there are certain advantages to transcendent powers, but takes his central thesis-that powers are intrinsic truthmakers for the laws of 
nature-to be compatible with immanence as well. ${ }^{29}$ Heil, on the other hand, rejects Platonism. ${ }^{30}$ For Heil, there are particular substances and particular modes-things, and ways things are. We can abstract away from a red tomato's shape, size, location, and so forth, to get to its redness, just as we can abstract away from its properties to get to the underlying substance, but abstractions do not exist in Plato's heaven; indeed, they do not really exist anywhere. It is unclear whether abstracting from particular ways of being provides us with mindindependent universals, but what is clear is that there are no uninstantiated powers in Heil's ontology. As Heil puts it, "Statements concerning universals can be true, but their truthmakers are wholly particular. Universals, as Armstrong would say, involve 'no addition to being." ${ }^{31}$ Take two red tomatoes. We can abstract away from all the other features of each tomato to get to their colours, and we find these properties to be exactly resembling. However, for Heil, exact resemblance as to colour does not require a shared universal, or any property over and above the particular ways of being coloured of the tomatoes.

Despite their unattractiveness to Aristotelians, transcendent powers can certainly secure the intrinsicality of powers against their relational individuation. Platonists typically hold that universals are abstract and exist at any possible world, regardless of whether they have instances there. On this view, for any power $\mathrm{P}$, there could be a lonely $\mathrm{P}$ at some world $\mathrm{W}$, because the other powers upon which $\mathrm{P}$ depends are bound to exist at $\mathrm{W}$. In one sense, of course, $\mathrm{P}$ is not lonely, because it is accompanied by the other powers in relation to which it is individuated. However, this is not the kind of accompaniment that would render $\mathrm{P}$ extrinsic. The key point is that given transcendent powers, a concrete particular need not be accompanied by any other concrete particulars in order to possess $\mathrm{P}$, which renders the relational individuation of powers consistent with their intrinsicality. Powers, on this view, are individuated by their places in an abstract relational structure, but are nonetheless intrinsic to their bearers, which need not stand in any relations in order to instantiate them. ${ }^{32}$

Perhaps there is a way for Aristotelians to square the intrinsicality of powers with their relational individuation without abandoning immanence. The key, one might suspect, lies in coinstantiation of powers. Roughly, for any powers $\mathrm{P}$ and $\mathrm{Q}$,

${ }^{29}$ Bird (2007a). $\quad{ }^{30}$ Heil (2012), ch. 5.

${ }^{31}$ Heil (2012), p. 104. Lowe also rejects Platonism on the grounds that universals are abstractions from particular modes of substances. Qua abstractions, universals are not located anywhereneither in Plato's Heaven, nor in their instances. As with Heil, is difficult to see how Lowe could allow for uninstantiated universals.

32 Bird (2007b). Bird holds that powers are the nodes of an asymmetric graph structure whose vertices are stimulus and manifestation relations, with the identities of the nodes determined by the structure. 
there will be a lonely $\mathrm{P}$ even if $\mathrm{P}$ depends on $\mathrm{Q}$ and there are no uninstantiated powers, provided there is a lonely $\mathrm{P}$ and $\mathrm{Q}$. For all their mutual ontological dependence, there does exist a lonely charge, and a lonely mass: it is a lonely electron. Electrons have both charge and mass, so provided neither property depends on any further property that electrons lack, the mutual dependence of charge and mass does not preclude their intrinsicality. In general, mutual dependence is consistent with intrinsicality and the rejection of Platonism provided any properties $\mathrm{P}_{1}, \ldots, \mathrm{P}_{\mathrm{n}}$ that depend on each other are possibly coinstantiated. Supposing for the sake of argument that mass and charge depend on each other and upon no other properties, the existence of electrons guarantees that both are intrinsic. There is at least one counterintuitive consequence of this theory. First, note that there are things that have mass but no charge-neutrinos, for example. ${ }^{33}$ If we need coinstantiation to explain how ontological dependence is consistent with intrinsicality, we are forced to say there could not be a lonely massive neutrino, because such a neutrino would need to exist at a world containing no charge to help individuate its mass, which violates the mutual dependency of mass and charge.

The counterintuitive consequence is that mass is an intrinsic power of neutrinos because there exists a lonely electron. Surely, one might object, if mass is intrinsic to this neutrino, then it is not enough that there exists a lonely mass-it should be possible for the neutrino to be that mass. However, if the above speculation on the mutual dependence of mass and charge is correct, then for a neutrino to have mass, something must be charged, and it cannot be the neutrino. Langton and Lewis's analysis implies, in the current context, that some intrinsic properties are such that not everything that has them could have them if lonely. Mass is basic intrinsic because it is a perfectly natural property that is independent of accompaniment-there is a lonely mass, which is also charged, therefore not a neutrino. If mass is an intrinsic property simpliciter, however, then the neutrino's mass is intrinsic to it, despite the fact that its having mass requires the existence of a numerically distinct charged particle. ${ }^{34}$

The trouble with this result is that we are inclined to think of intrinsic properties as those that characterize the way its bearers are, independently of

\footnotetext{
33 The three kinds of neutrino recognized as fundamental particles in the standard model each have non-zero mass and zero electric charge. I speak of neutrinos here rather than neutrons because neutrons are composed of three quarks, each having fractional electric charges that add up to zero. Wherever neutrons exist, something is charged, but the common charged particulars are proper parts of the neutron and so not distinct from it.

${ }^{34}$ Have I not assumed, in the preceding argument, that neutrinos themselves could not possibly be charged? Yes, but no matter. It is equally counterintuitive to suppose that a neutral neutrino having its mass intrinsically depends on the possibility of a charged neutrino.
} 
anything else. If mass can only be instantiated by an accompanied neutrino, then what does it matter that some possibly lonely things, such as electrons, have it? In contrast to Langton and Lewis's account, all three alternative accounts of intrinsicality discussed earlier in section 9.2 imply that mass is extrinsic, under our present assumptions. According Witmer, Butchard, and Trogdon, mass will come out extrinsic, because a neutrino has mass partly in virtue of being accompanied by something charged, and being so accompanied is not independent of accompaniment, so something massive-a neutrino-fails to have mass in an intrinsic fashion. Vallentyne's account has the same consequence. According to his theory, if mass is intrinsic, then anything massive would retain its mass if everything else were taken away. Neutrinos fail this test. Further, since neutrinos have mass partly in virtue of standing in the relation of being accompanied to something charged, Francescotti's theory also entails that mass is extrinsic.

The moral is that for Aristotelians, whatever powers a given power $\mathrm{P}$ ontologically depends upon had better be powers that all possible bearers of $\mathrm{P}$ possess; that something has both the dependent power and the power upon which it depends is not sufficient. We can still render this sort of dependency consistent with immanent universals, however, provided we strengthen the coinstantiation thesis so that powers same-subject metaphysically necessitate all other powers upon which they depend. For charge to be the property it is, mass must exist for individuative purposes. If in addition (i) charge is intrinsic, (ii) there are no uninstantiated properties, and (iii) nothing has its intrinsic properties in virtue of anything else, then (iv) charge must same-subject necessitate mass. The empirical fact that we never observe massless charges may be a consequence of this deeper metaphysical dependency.

Unfortunately, this theory implies that mass does not depend upon charge, because although we do not see any massless charges in nature, we see plenty of neutral masses-the neutrino being an example. We have solved the problem posed by the lonely neutrino by denying that mass depends on charge. Perhaps this is true, but pause for a moment to reflect on the consequences if it is. I suggested in section 9.3 that we could read off ontological dependencies from fundamental laws, but if the current theory is correct, this is not so. The Schrödinger equation for a particle moving in an electromagnetic field suggest that mass and charge are mutually ontologically dependent, but they are clearly not mutually necessitating. Whichever power mass is, it cannot be the power to do all that Schrödinger's equation says it does. ${ }^{35}$ If it were, then mass would ontologically depend on charge, which, given the strong version of the

35 The same argument applies, mutatis mutandis, to any laws relating mass to charge. 
coinstantiation thesis, means there could be no neutral masses. Since there are neutral masses, the way in which mass interacts with charge cannot be individuative of mass.

The only way I can see to avoid this sort of result, holding on to relational individuation, immanence, and intrinsicality, is to reject the idea that loneliness is a necessary condition for intrinsicality. On this view, a power $\mathrm{P}$ is intrinsic to its bearers and yet requires the existence of other particulars as bearers of any powers upon which $\mathrm{P}$ ontologically depends. Consider again the neutrino, which cannot be lonely on the assumption that mass ontologically depends on charge. The fact that neutrino $x$, in order that its mass $m$ be individuated, requires the existence of some particle $y$ with some charge $q$, stems not from the fact that $x$ 's having mass is a property that somehow involves $x$ standing in a relation to $y$, but from the fact that $m$ is partially individuated by certain dispositional relations to $q$. Perhaps the right thing to say is that loneliness is a suitable necessary condition for intrinsicality only if one is Humean about causality; for Aristotelians, a different account is needed. I will not take on the challenge of supplying such an account here, because none of the proposed ways of reconciling relational individuation with intrinsicality, I shall now argue, is of use to the Aristotelian who wishes to argue that causal relations are not genuine relational properties.

\subsection{Conclusion: The Irreducibility of Causality to Powers}

In section 9.4, I considered two notions of self-contained powers: (a) power manifestations as further states of the powers themselves; (b) powers as accidentally the powers they are, but essentially ineffable. I argued that (a) failed to apply to physical properties such as mass and charge, and that (b) was not really a theory of powers at all. In section 9.5, I considered three ways of reconciling the relational individuation of powers with their intrinsicality: (i) powers as transcendent universals; (ii) powers as dependent only on properties they samesubject necessitate; (iii) powers as intrinsic on some understanding of 'intrinsic' that does not require loneliness. I consider (iii) to be by far the most promising of these options, but set that aside. Securing intrinsicality in any one of these ways may be sufficient to show that token causal relations are internal, but not that causation itself is reducible.

That Alf is $0.1 \mathrm{~m}$ taller than Bob is an internal relation, requiring no relational property to be instantiated by the ordered pair (Alf, Bob). 'Alf is $0.1 \mathrm{~m}$ taller than Bob' is made true by the intrinsic heights of Alf and Bob, and so is not an irreducibly relational state of Alf and Bob. Arguing against the reducibility of 
asymmetric transitive relations in general, Russell pointed out that in order to argue this way, we must still posit a relation between the magnitude of Alf's height and the magnitude of Bob's height. ${ }^{36}$ The case for the internality of taller than rests depends on arithmetical relations, in this case the fact that 1.85 is 0.1 greater than 1.75, which is a further asymmetric transitive relation. This does not mean that Alf's being $0.1 \mathrm{~m}$ taller than Bob involves Alf and Bob standing in an irreducible, external relation. However, if Russell is correct, we will not be able to apply this strategy to show that all relations are internal, for it is not at all obvious that the greater than relation between magnitudes can be treated in the same way as the taller than relation between Alf and Bob. There are, it seems, no intrinsic properties of magnitudes in virtue of which they stand in the relations they do.

All is not lost in the case of taller than, which we have still reduced to a more fundamental greater than relation between magnitudes. In the case of powerful causation, I submit, things are not so straightforward. While there are ways to reconcile the relational individuation of powers with their intrinsicality, they all presuppose individuative type-level relations between the powers themselves. The prospects for a reduction of causation begin to look bleak when one reflects on the fact that the individuative relations posited are type-level correlates of the token causal relations we were trying to reduce. Those who treat powerful causation as an internal relation must endorse some form of powers theory of causation. According to one such theory, causes are token comings together of reciprocal powers, effects their token mutual manifestations. However, once we allow that those powers are individuated by their potential for combining with other powers and manifesting in this way, then we succeed in reducing token causal relations only by presupposing type-level relations of the same kind.

This is certainly a reduction, but it is important to note the kind of reduction it is - of actual causal relations to potential causal relations, not of causality itself. It is not clear, unless one is prepared to deny that powers are relationally individuated at all, that we can eliminate all causal relations by embracing a powers theory. Perhaps one might suggest that the individuative structural relations between powers are internal. Well, what could that mean? Certainly not that such relations are determined by intrinsic properties of the powers, for in that case (i) we should have to posit second-order properties of powers, and (ii) the question would arise as to how those properties ground the first-order relations, and the same problem would simply recur at the second-order, leading to a regress. There are other notions of internality available, for instance Bradley's idea that internal relations are essential to the relata, and this seems much closer 
to the truth about relational individuation. ${ }^{37}$ If relations that are internal in this sense are also somehow reducible in that they do not require relational properties as truthmakers, then the reductionists are home and dry. Let us therefore briefly consider whether relations that are internal in this second sense are reducible.

Consider the following three grades of internal relatedness, focusing on the taller than relation. First case. Alf and Bob are contingently $1.85 \mathrm{~m}$ and $1.75 \mathrm{~m}$ tall respectively. Necessarily, if both Alf and Bob exist and have their actual intrinsic properties, Alf is taller than Bob. Second case. Suppose in addition that Alf and Bob have their heights essentially, and that all essential properties are necessary. In this case, necessarily, if both Alf and Bob exist, Alf is taller than Bob. It does not follow that they are essentially related, because either could exist without the other. In both the first and second cases, taller than is plausibly an internal relation in the sense that it is determined by the intrinsic properties of the relata, and hence is reducible. Third case. To get Bradleyan internality, we need to suppose that it is part of the essential nature of both Alf and Bob that Alf is taller than Bob. It does not follow that Alf and Bob have their heights essentially, as there are indefinitely many ways for Alf to be taller than Bob. What follows now is that necessarily, wherever either Alf or Bob exists, they both exist, and are such that Alf is taller than Bob. This, I take is, is analogous to the ontological dependence that results from the relational individuation of powers.

Now it is tempting to suppose that even in the third case, taller than is internal in both Bradley's essentialist sense and in the sense required by the reductionist, but this is a mistake. If Alf and Bob are essentially such that Alf is taller than Bob, then their heights are (arguably) not intrinsic. It is metaphysically impossible for Alf to be $1.85 \mathrm{~m}$ tall at worlds where Bob is $1.9 \mathrm{~m}$ tall, for instance. In order to have any particular height, Alf must first stand in the taller than relation to Bob. Neither can have a particular height independently of the height of the other, because neither can exist unless they stand in the appropriate relative height relation. This of course sounds wildly implausible, but that is just because concrete particulars are not essentially related by relations such as taller than. If they were, it would follow that height were not intrinsic, due to the ontological priority of relative height. My point here is that the cost of a relational essence is the metaphysical fundamentality of the individuating relations. If a given relation is ontologically prior to its relata, then it can be treated as neither determined by, nor reducible to, their intrinsic properties.

\footnotetext{
${ }^{37}$ Bradley (1897). See, for instance, his claim at p. 347 that a relation "essentially penetrates the being of its terms." For more on Bradley's view that relations are internal in this sense, see Ch. 1.3 in this volume.
} 
It is controversial whether relations could be prior to their relata, and one might suspect an incoherence in the very idea. In an ontology of pure powers, writes Lowe:

$[\mathrm{N}] \mathrm{o}$ property can get its identity fixed, because each property owes its identity to another, which, in turn owes its identity to another-and so on, in a way that, very plausibly, generates either a vicious infinite regress or a vicious circle. ${ }^{38}$

I am inclined to agree, but the problem Lowe raises targets only the claim that powers are wholly individuated by their relations to each other. It seems clear that we can solve Lowe's regress problem by allowing some non-powers into the ontology-that is, by allowing that some of the properties in the relational structure that individuates powers have their identities determined independently of their own places in that structure. Bird agrees:

If we trace the chain of dependencies, from the essence of one essentially dispositional property to another, we will eventually come across essentially dispositional properties whose manifestations or stimuli are characterized not in terms of yet further essentially dispositional properties but in categorical terms instead. Since these have their identities primitively, they serve to determine the identities of all the properties in the structure. ${ }^{39}$

The issue of the reducibility of causation does not turn on whether or not powers need type-level relations to both powers and non-powers in order to be properly individuated. The ontological primacy of relations remains, whether the ontology is pure or mixed; all that changes are the natures of (some of) the relata. Put differently: what is at stake in the debate over the coherence of pure powers ontologies is not whether structure could be ontologically basic, but whether everything ontologically basic could be structure. In any powers ontology, I submit, type-level causal relations to other properties-whether or not these latter are individuated outside the structure or within it-will be ontologically prior to the powers, hence irreducible. Powerful causation reduces token-causal relations to intrinsic powers only at the expense of the irreducible type-level causal relations needed to individuate the powers. Absent a plausible theory of self-contained powers, powerful causation does not make good on its promise to provide non-relational truthmakers for all the causal truths. ${ }^{40}$

${ }^{38}$ Lowe (2006a), p. 138. I have assumed a finite set of mutually dependent properties here for simplicity, but an infinite regress of ontological dependencies, as Bird argues in his (2007b), is no better than a circle. See Ladyman (Ch. 11 in this volume) for a defence of purely relational individuation in ontic structural realism.

39 Bird (2007b), p. 526.

${ }^{40}$ Based on research funded by the European Research Council. Early versions of some of the arguments of this paper were presented, on two separate occasions, at Oxford. Thanks to John Heil, Ross Inman, Jonathan Lowe, Brian Prince, Célia Teixeira, and Alastair Wilson for helpful comments and criticisms. 